Ann. Biol. anim. Bioch. Biophys., I973, 13 (3), 38I-397.

\title{
INFLUENCE DU NIVEAU ET DE LA NATURE DE L'APPORT ALIMENTAIRE DE MAGNÉSIUM SUR LE MÉTABOLISME DU PHOSPHORE DU CALCIUM ET DU MAGNÉSIUM CHEZ LE RAT EN CROISSANCE
}

\author{
A. POINTILLART et L. GUÉGUEN \\ avec la collaboration technique de Colette CoLIN \\ Station de Recherches de Nutrition, \\ Centre national de Recherches zootechniques, I. N.R.A., \\ 78350 Jouy en Josas \\ RÉSUMÉ
}

Les expériences ont porté sur des rats mâles en croissance recevant un apport supplémentaire de magnésium variant par la quantité (de I,5 à 8,o g par $\mathrm{kg}$ de matière sèche) ou par la nature du sel (chlorure, sulfate, carbonate, oxyde, hydroxyde, lactate) et dont le régime contenait ro g de $\mathrm{Ca}$ et $8 \mathrm{~g}$ de $\mathrm{P}$ par $\mathrm{kg}$ de matière sèche.

Par des bilans simples de to jours ou des bilans de radioactivité $\left({ }^{45} \mathrm{Ca}\right.$ ou $\left.{ }^{32} \mathrm{P}\right)$ les différents paramètres du métabolisme de $\mathrm{Ca}, \mathrm{P}$ et $\mathrm{Mg}$ ont été évalués.

La surcharge alimentaire en $\mathrm{Mg}$, quel que soit le sel administré, provoque une hypophosphaturie très nette, une diminution de l'absorption apparente du phosphore, une augmentation des pertes fécales endogènes de $P$ et, dans certains cas (avec le chlorure, le sulfate et le lactate), du calcium urinaire. Le carbonate de magnésium a diminué l'absorption du calcium tandis que le chlorure l'a augmentée. Certains rats ayant reçu l'oxyde de magnésium ont formé des calculs urinaires.

Il ne semble pas se produire de compétition entre calcium et magnésium au niveau de l'absorption intestinale.

La rétention et l'excrétion urinaire de calcium semblent dépendre davantage de l'apport anionique ou de l'équilibre acido-basique que de l'apport du supplément de magnésium.

\section{INTRODUCTION}

Il existe des interactions nombreuses (LARvor et DURLACH, I97I $b$ ) entre le magnésium et les autres composants du régime, notamment le calcium et le phosphore (PonTTILIART, I97I). AlcoCk et MCINTYRE (Ig62), en privant totalement des rats de magnésium, constatent, après avoir obtenu les signes cliniques de la carence, que le calcium est mieux absorbé par le tube digestif lorsque le magnésium 
est absent dans le régime. CLARK et BELANGER (1967) reprochent à ces deux auteurs d'avoir travaillé dans des conditions pathologiques et entreprennent une série d'expériences mettant en jeu des apports très variables de calcium, de phosphore et de magnésium. Les résultats montrent que le magnésium en excès dans le régime augmente l'excrétion fécale de phosphore, diminue celle du calcium, augmente l'excrétion urinaire du calcium et diminue celle du phosphore. Les travaux de Clark (Clark, I969 $a$, $b$, et $c$, I968 $a$ et $b$; Clark et RIVERA-CoRdero, I97I) portaient sur les effets de la supplémentation en magnésium sous forme de chlorure, chez des rats adultes, sur les bilans apparents de $\mathrm{Ca}, \mathrm{P}$ et $\mathrm{Mg}$. Compte tenu des travaux de RaNDorn et al. (1952) sur l'influence de l'anion associé au magnésium et de l'absence quasi totale (BRISCOE et RAGAN, 1966) d'études portant sur l'influence du magnésium sur l'excrétion fécale endogène de $\mathrm{Ca}$ et $\mathrm{P}$, il nous a semblé utile d'étudier, chez des rats en croissance, l'influence de la supplémentation en $\mathrm{Mg}$ et le rôle éventuel de l'anion associé, non seulement par des méthodes de bilans simples, mais également à l'aide de techniques radioisotopiques.

\section{MATÉRIEL ET MÉTHODES}

\section{A. - Animaux et régimes alimentaires}

Toutes les expériences ont porté sur des rats mâles albinos de souche Wistar pesant de I 30 à I6o $\mathrm{g}$ chacun au moment de la mise en lots et âgés de 6 semaines environ, maintenus en cages individuelles à métabolisme permettant de récolter séparément l'urine et les fèces. Le régime ali-

TABLEAU I

Composition des aliments

\begin{tabular}{|c|c|}
\hline \multicolumn{2}{|l|}{ Formule de l'Aliment de base (p. 100) } \\
\hline Blé $\ldots \ldots \ldots \ldots \ldots \ldots \ldots$ & 70 \\
\hline Son $\ldots \ldots \ldots \ldots \ldots \ldots$ & 4 \\
\hline Farine de poisson .......... & 12 \\
\hline Farine de viande $\ldots \ldots \ldots \ldots$ & $\mathbf{5}$ \\
\hline Huile de mais...$\ldots \ldots \ldots$ & 6 \\
\hline Composé minéral . & 3 \\
\hline \multicolumn{2}{|l|}{ Formule du composé minéral (p. 100) } \\
\hline Phosphate bicalcique..$\ldots$ & 32,57 \\
\hline Chlorure de sodium ... & 23,80 \\
\hline Chlorure de potassium..... & 12,40 \\
\hline Hydroxyde de magnésium. & 15,00 \\
\hline Phosphate monopotassique & 10,00 \\
\hline Sulfate de fer $\ldots \ldots \ldots \ldots$. & 5,47 \\
\hline Sulfate de manganèse . & 0,06 \\
\hline Sulfate de cuivre .... & 0,04 \\
\hline Sulfate de zinc... & 0,10 \\
\hline Sulfate de cobalt $\ldots \ldots \ldots$ & 0,07 \\
\hline Iodure de potassium stabilis & 0,40 \\
\hline
\end{tabular}

Un mélange vitaminique contenant toutes les vitamines indispensables apportait notamment $500 \mathrm{UI}$ de vitamine $\mathrm{D}_{3}$ et $1000 \mathrm{UI}$ de vitamine $\mathrm{A}$ par $\mathrm{kg}$ d'aliment. 
mentaire, dont la composition figure dans le tableau $I$, était distribué aux rats sous forme de bouillie à raison de I $\mathrm{kg}$ d'aliment pour I,2 1 d'eau distillée.

L'alimentation était semi ad libitum et, dans les derniers essais portant sur les différents sels de magnésium, l'alimentation des témoins était restreinte sur la base de la consommation des rats supplémentés en $\mathrm{Mg}$. De l'eau distillée était distribuée à volonté.

La ration de base (tabl. I) apportait au départ Io $\mathrm{g}$ de calcium et $8 \mathrm{~g}$ de phosphore par $\mathbf{k g}$ d'aliment sec. Les teneurs en magnésium suivantes ont été choisies : 1,$5 ; 2 ; 3.5 ; 4 ; 7$ et $8 \mathrm{~g}$ par $\mathrm{kg}$ de matière sèche (MS) ce qui a permis de doubler ( 2 et $4 ; 3,5$ et 7 ), de tripler ( 2 et 6 ) ou de quadrupler ( 2 et $8 ; 1,5$ et 6 ) la teneur en magnésium du régime témoin, en partant d'une teneur de base $(\mathrm{r}, 5 ; 2 ; 3,5)$ proche ou éloignée du besoin minimum.

Le but des essais entrepris était d'étudier les effets de la supplémentation en Mg en faisant varier, soit la teneur en $\mathrm{Mg}$ du régime par addition de chlorure de magnésium $\left(\mathrm{MgCl}_{2}, 6_{2} \mathrm{HO}\right)$, soit la source d'apport (chlorure, sulfate, carbonate, lactate, oxyde et hydroxyde de magnésium).

Tous les essais comprenaient une période de bilan classique de ro jours précédée d'une période d'adaptation de 12 jours environ. A l'issue de ces bilans, certains animaux ont reçu, soit par voie orale, soit par voie intrapéritonéale, une dose de radioélément qui permettait de déterminer, avec la méthode des bilans comparés pour le calcium, la méthode de dilution isotopique pour le phosphore, quelles étaient les pertes endogènes fécales de $\mathrm{Ca}$ ou de $\mathrm{P}$.

Chacun des quatre premiers essais a porté sur I6 rats répartis en 2 lots de 8 . Les deux essais portant sur les divers sels de $\mathrm{Mg}$ ont été effectués respectivement sur $\mathrm{I} 6$ rats répartis en 4 lots de 4 et sur 20 rats répartis en 5 lots de 4 .

\section{B. - Prélèvement et analyse des échantillons}

Durant les périodes de bilans, les quantités consommées en matière fraîche et en matière sèche, et les quantités excrétées étaient mesurées chaque jour.

Après minéralisation au four à $55^{\circ} \mathrm{C}$ d'une prise d'essai d'aliment, de fèces, d'urine ou de plasma (préalablement séchées) et mise en solution acide, le phosphore a été dosé à l'aide de la méthode colorimétrique au phosphovanadomolybdate d'ammonium (réaction de Misson), le calcium par photométrie de flamme (photomètre Eppendorf), le magnésium par spectrométrie d'absorption atomique (appareil Hilger et Watts) et les mesures de radioactivité ont été faites par scintillation liquide pour ${ }^{45} \mathrm{Ca}$ (appareil Nuclear Chicago) suivant la technique décrite par Patterson et Greene (1965), ou à l'aide d'un compteur Geiger-Müller (appareil Tracerlab) pour ${ }^{32} \mathrm{P}$.

\section{C. - Méthodes de calcul des paramètres du métabolisme du phosphore et du calcium}

Pour le phosphore, la proportion de $\mathrm{P}$ endogène dans $\mathrm{P}$ fécal total a été obtenue par la méthode de dilution isotopique de LofGREen et KLEIBER (1954) déjà décrite par GUEGUEN (I962, 1963) dans le cas du phosphore. Cette proportion est égale au rapport moyen des activités spécifiques du phosphore dans les fèces du $7^{\mathrm{e}}$ au ${ } 6^{\mathrm{e}}$ jour après l'injection et du phosphore dans les urines du $6^{\mathrm{e}}$ au $15^{\mathrm{e}}$ jour.

Pour le calcium, compte tenu des difficultés de mesure de la radiaoctivité spécifique du calcium urinaire, nous avons utilisé la méthode des bilans comparés décrite par HANSARD et al. (1952) et Comar et al. (I953). Ainsi, nous avons mesuré le coefficient d'utilisation digestive réelle (CUDr) de ${ }^{45} \mathrm{Ca}$ puis, connaissant le CUD apparent du calcium alimentaire obtenu par le bilan simple, nous en avons déduit la perte fécale endogène de calcium.

\section{RÉSULTATS}

\section{A. - Influence de l'apport alimentaire de magnésium sous la forme de chlorure de magnésium}

\section{a) Croissance et consommation.}

Pour l'ensemble des animaux, la vitesse de croissance n'est jamais descendue en dessous de $5 \mathrm{~g}$ par jour. Les différences entre rats témoins et supplémentés, quand elles apparaissent, sont relativement peu importantes, les modifications les plus 
grandes ayant été constatées chez les rats recevant $8 \mathrm{p}$. I ooo de $\mathrm{Mg}$ dans le régime sec. Ainsi, les rats témoins recevant $2 \mathrm{p}$. $\mathrm{x}$ ooo de $\mathrm{Mg}$ ont consommé quotidiennement $20 \mathrm{~g}$ d'aliment $\mathrm{sec}$, ont eu un gain de poids de $8 \mathrm{~g}$ par jour et ont utilisé la matière sèche du régime à raison de $85 \mathrm{p}$. Ioo environ, tandis que les rats supplémentés à 8 p. I ooo de $\mathrm{Mg}$ n'ont consommé que I5 $\mathrm{g}(\mathrm{P}<\mathrm{O}, \mathrm{OI})$ d'aliment sec, la vitesse de croissance étant réduite à $5 \mathrm{~g}$ par jour $(\mathrm{P}<0, \mathrm{oI})$ et l'utilisation de la matière sèche du régime à $8 \mathrm{I}$ p. IO0 $(\mathrm{P}<0,0 \mathrm{r})$.

Les fèces des animaux supplémentés en $\mathrm{Mg}$ étaient plus molles que celles des témoins, ce qui, dans certains cas, notamment pour les rats recevant $8 \mathrm{p}$. r ooo de $\mathrm{Mg}$, a entraîné des diarrhées ayant causé la mort d'un rat. Il n'y a pas eu de diurèse accrue de façon significative chez les animaux dont le régime était enrichi en $\mathrm{Mg}$.

b) Métabolisme de Ca, $\mathrm{P}$ et $\mathrm{Mg}$.

Les résultats moyens des bilans de $\mathrm{Ca}, \mathrm{P}, \mathrm{Mg}$ sont rassemblés dans les tableaux 2, 3 et 4 .

Dans les essais A et B (tabl. 2 et 3), la consommation journalière de phosphore et de calcium ayant été diminuée chez les animaux supplémentés en $\mathrm{Mg}$, l'interprétation statistique a été limitée aux seules différences moyennes exprimées en $\mathrm{p}$. Ioo de l'ingéré. Dans l'essai C, par contre, les témoins ayant été restreints, tous les animaux ont consommé la même quantité de calcium et de phosphore.

Au niveau urinaire, la supplémentation en magnésium, sous forme de chlorure, augmente l'excrétion de calcium de façon très significative $(P<0,0 I)$ dans les essais $B$ et $C$ tandis qu'elle diminue de façon très significative $(P<0,0 I)$ l'excrétion du phosphore dans les trois essais (tabl. 5 et 6 ).

Au niveau digestif, l'absorption du calcium, en p. Ioo de l'ingéré, augmente $(\mathrm{P}<0,0 \mathrm{r})$ pour les animaux recevant 6 et $8 \mathrm{p}$. I ooo de $\mathrm{Mg}$ tandis que l'absorption du phosphore diminue $(\mathrm{P}<0,0 \mathrm{I})$ chez tous les rats recevant un supplément de $\mathrm{Mg}$.

L'excrétion fécale endogène de calcium n'est pas modifiée (tabl. 7) par la supplémentation en magnésium sous forme de chlorure, alors que l'excrétion urinaire de calcium passe de 0,4 (témoin) à 3,I p. Ioo de l'ingéré (supplémentés). Par contre, la perte fécale endogène de phosphore (tabl. 8) augmente avec le magnésium alimentaire.

Les conséquences au niveau du bilan résultent des faits relevés aux niveaux intestinal et urinaire. L'amélioration de la rétention du calcium (en p. roo de l'ingéré) constatée dans les lots recevant 6 et $8 \mathrm{p}$. I ooo de $\mathrm{Mg}$ est due surtout à une meilleure absorption, tandis que l'amélioration de la rétention du phosphore constatée chez tous les rats recevant un supplément de $\mathbf{M g}$ est principalement due à une élimination urinaire considérablement diminuée.

\section{B. - Infuence de la nature de l'apport alimentaire de $\mathrm{Mg}$}

a) Excrétion urinaire et endogène fécale de $\mathrm{Ca}, \mathrm{P}$ et $\mathrm{Mg}$.

Les effets de la surcharge en $\mathrm{Mg}$ sur la calciurie dépendent beaucoup de l'anion associé à $\mathrm{Mg}$. Le chlorure et le sulfate (tabl. 5) et, dans une moindre mesure, le lactate (tabl. 6) augmentent le calcium urinaire tandis quel'oxyde, l'hydroxyde et le carbonate n'ont pas d'influence (tabl. 6). 


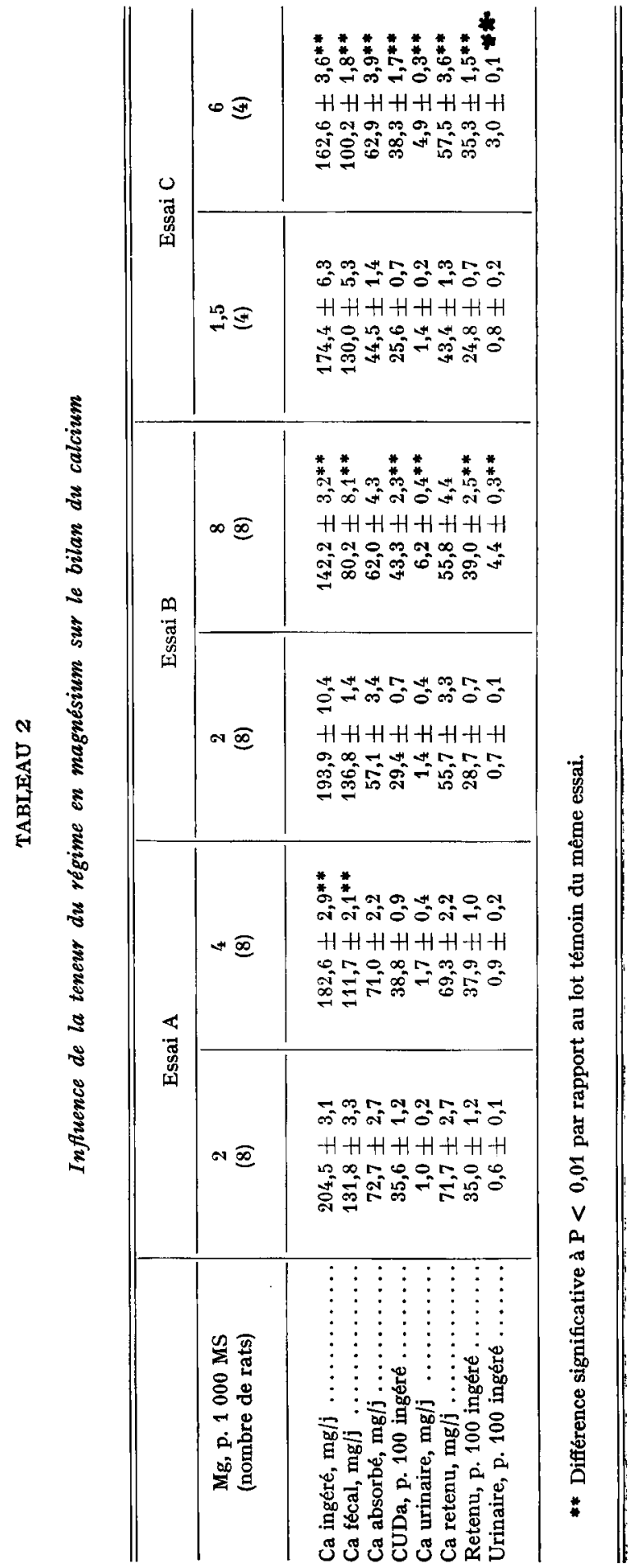


A. POINTILLART, L. GUÉGUEN

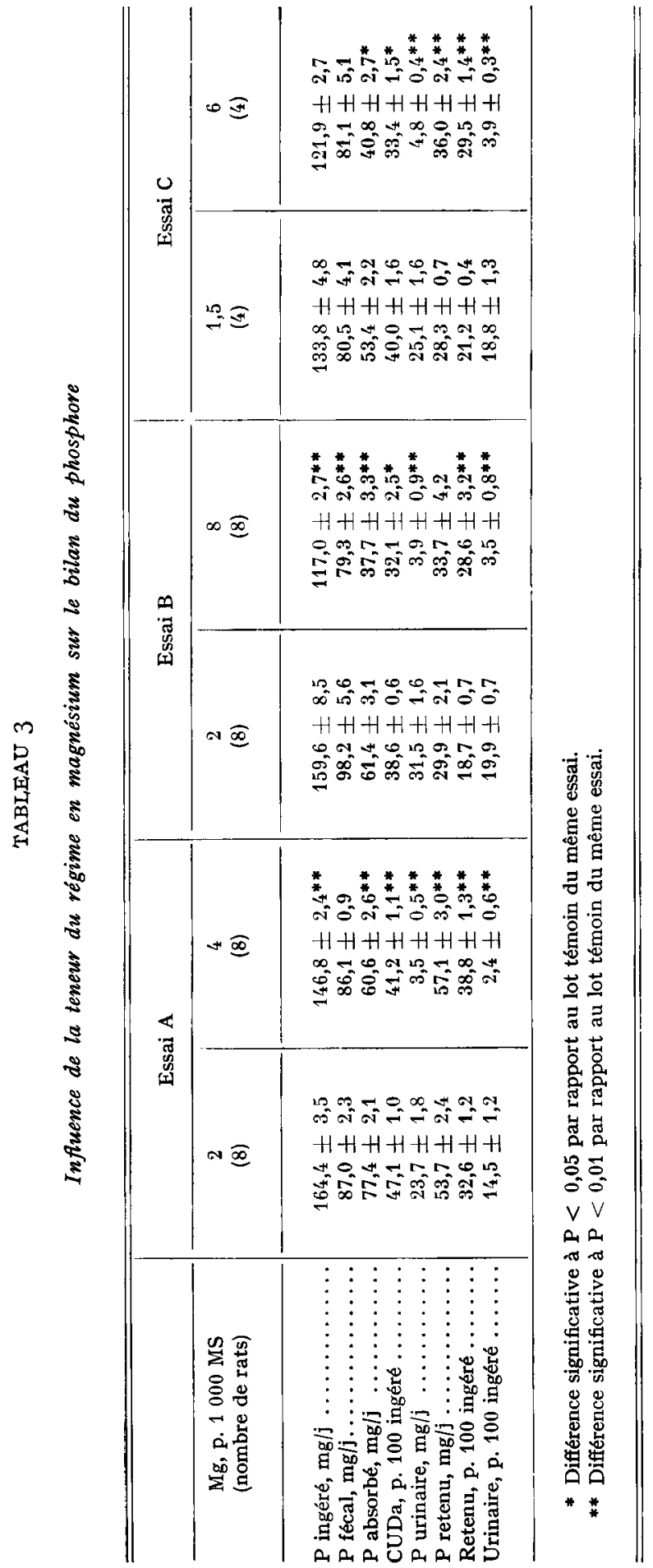




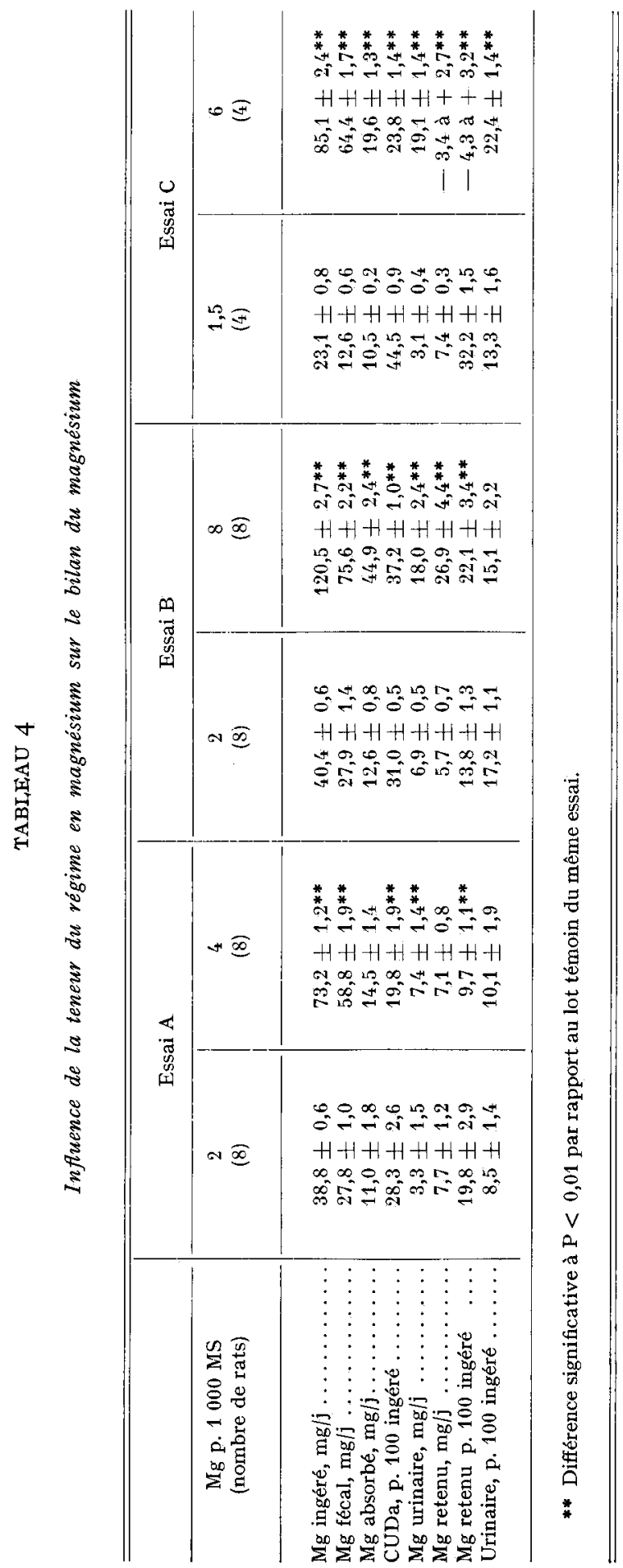




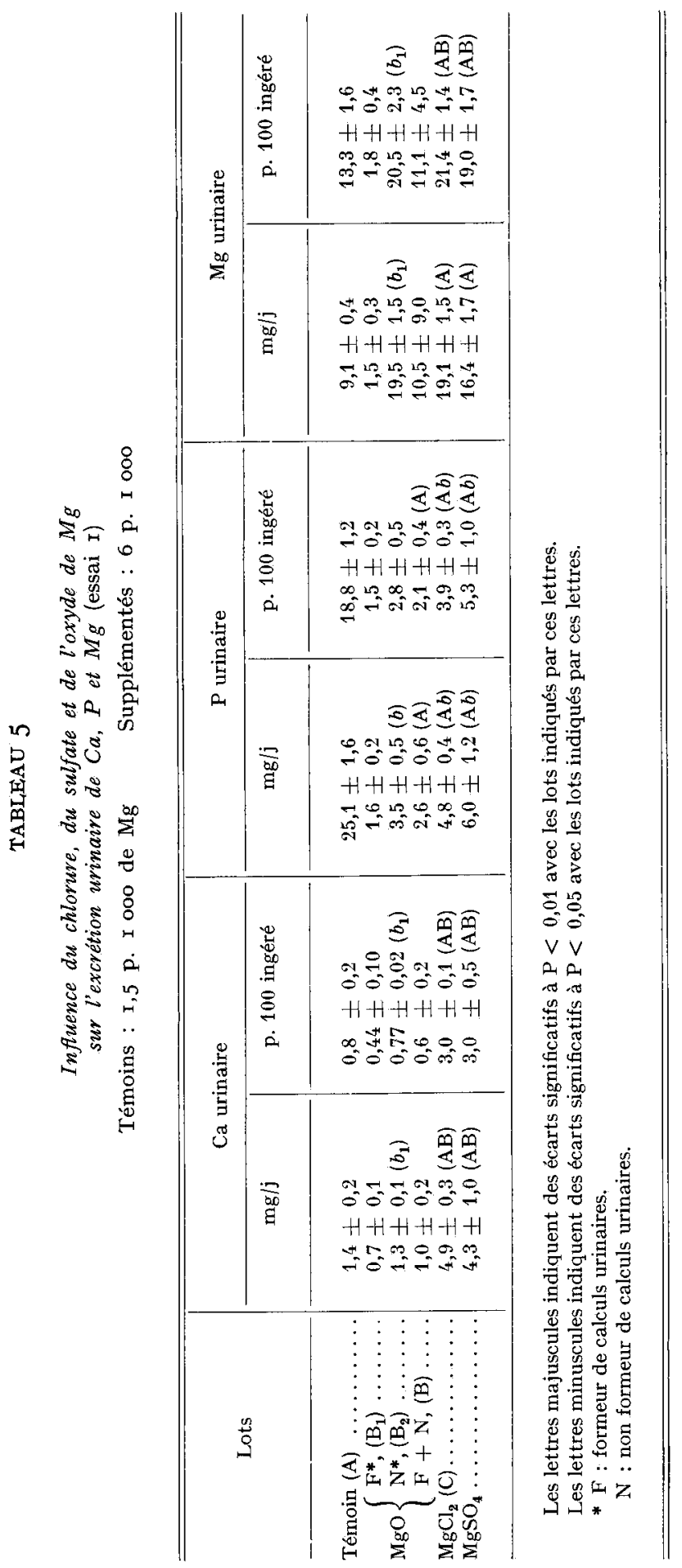




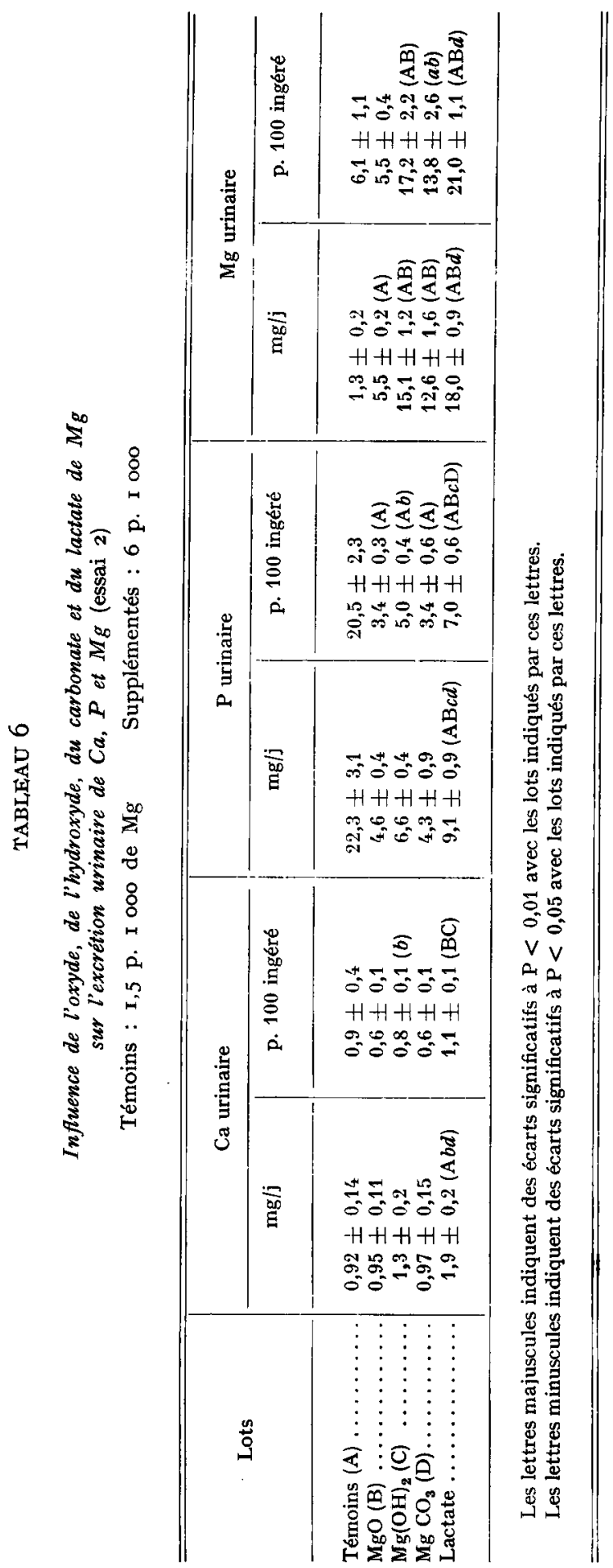


Le magnésium en excès dans le régime diminue considérablement la phosphaturie quel que soit l'anion (tab1. 5 et 6 ).

Avec toutes les formes d'apport, mis à part l'oxyde de magnésium, la magnésiurie augmente considérablement.

Il existe un parallélisme entre les variations des excrétions urinaires de $\mathrm{Ca}, P$ et $\mathrm{Mg}$ provoquées par un même anion. Ainsi, avec le chlorure et le sulfate (tabl. 5) les excrétions de $\mathrm{Ca}, \mathrm{P}$ et $\mathrm{Mg}$ sont supérieures à celles constatées avec l'oxyde de magnésium. De même, avec le lactate (tab1.6) 1'excrétion urinaire des trois éléments est supérieure à celle obtenue avec le carbonate et l'oxyde de $\mathrm{Mg}$, tandis que les différences ne sont pas toujours significatives avec l'hydroxyde de $\mathrm{Mg}$.

\section{TABLEAU 7}

Influence de la surcharge en $\mathrm{Mg}\left(\mathrm{MgCl}_{2}\right)$ sur l'excrétion endogène fécale de Ca (méthode des bilans comparés avec ${ }^{45} \mathrm{Ca}$ )

\begin{tabular}{|c|c|c|}
\hline & $\begin{array}{l}\text { Lot témoin (8 rats) } \\
2 \text { p. } 1000 \text { de } \mathrm{Mg}\end{array}$ & $\begin{array}{c}\text { Lot supplémenté } \\
\text { (8 rats) } \\
6 \text { p. } 1000 \text { de } \mathrm{Mg}\end{array}$ \\
\hline $\begin{array}{l}\text { Ca ingéré, } \mathrm{mg} / \mathrm{j} \ldots \ldots \ldots \ldots \\
\text { Ca endogène, } \mathrm{mg} / \mathrm{j}\left({ }^{1}\right) \ldots \ldots \ldots \\
\text { Endogène, } \mathrm{p} .100 \text { ingéré }\left({ }^{1}\right) \ldots \ldots \\
\text { Ca exogène, } \mathrm{mg} / \mathrm{j}\left({ }^{1}\right) \ldots \ldots \ldots \ldots \\
\text { CUDa, p. } 100 \text { ingéré } \ldots \ldots \ldots \ldots \\
\text { CUDr, p. } 100 \text { ingéré }{ }^{(1)} \ldots \ldots \ldots\end{array}$ & $\begin{array}{l}217,6 \pm 3,5 \\
79,6 \\
36,6 \\
62,9 \\
34,5 \pm 1,5 \\
71,1\end{array}$ & $\begin{array}{l}187,5 \pm 4,7 \\
67,5 \\
36,0 \\
60,4 \\
31,9 \pm 1,2 \\
67,9\end{array}$ \\
\hline
\end{tabular}

(1) La méthode ne permet pas le calcul de l'écart-type.

Comme on peut le voir (tabl. 5) le lot MgO du premier essai a été divisé en deux autres lots $\mathrm{F}$ et $\mathrm{N}$, sur la base de l'excrétion urinaire de $\mathrm{Mg}$, en tenant compte du fait que dans le lot $F$ un rat est mort après avoir formé des calculs urinaires. Le lot $\mathrm{F}$ (formeur de calcul) se distingue du lot $\mathrm{N}$ (normal) par une excrétion significativement $(\mathrm{P}<0,05)$ inférieure de calcium, de phosphore et de magnésium.

Comme avec le supplément de chlorure de magnésium, l'excrétion endogène fécale de phosphore augmente (tabl. 8) avec un supplément d'oxyde et surtout de sulfate de magnésium.

b) Absorption intestinale et rétention de $\mathrm{Ca}, \mathrm{P}$ et $\mathrm{Mg}$.

Dans l'essai I (tabl. 9) les rats ayant reçu un supplément de chlorure de $\mathrm{Mg}$ ont absorbé relativement mieux le calcium et le phosphore que les autres rats. Toutefois, quel que soit l'anion utilisé, le CUDa du phosphore des rats supplémentés reste inférieur à celui des rats témoins, tandis que le CUDa du calcium peut devenir supérieur, avec le chlorure de magnésium notamment. La plus forte diminution du CUDa de $\mathrm{P}$ est obtenue avec le sulfate de magnésium. Le CUDr de $\mathrm{P}$ du régime supplémenté devient proche de celui de $\mathrm{P}$ du régime témoin pour le chlorure, mais reste inférieur pour l'oxyde et le sulfate. Dans l'essai 2 (tabl. 9), les rats ayant reçu 


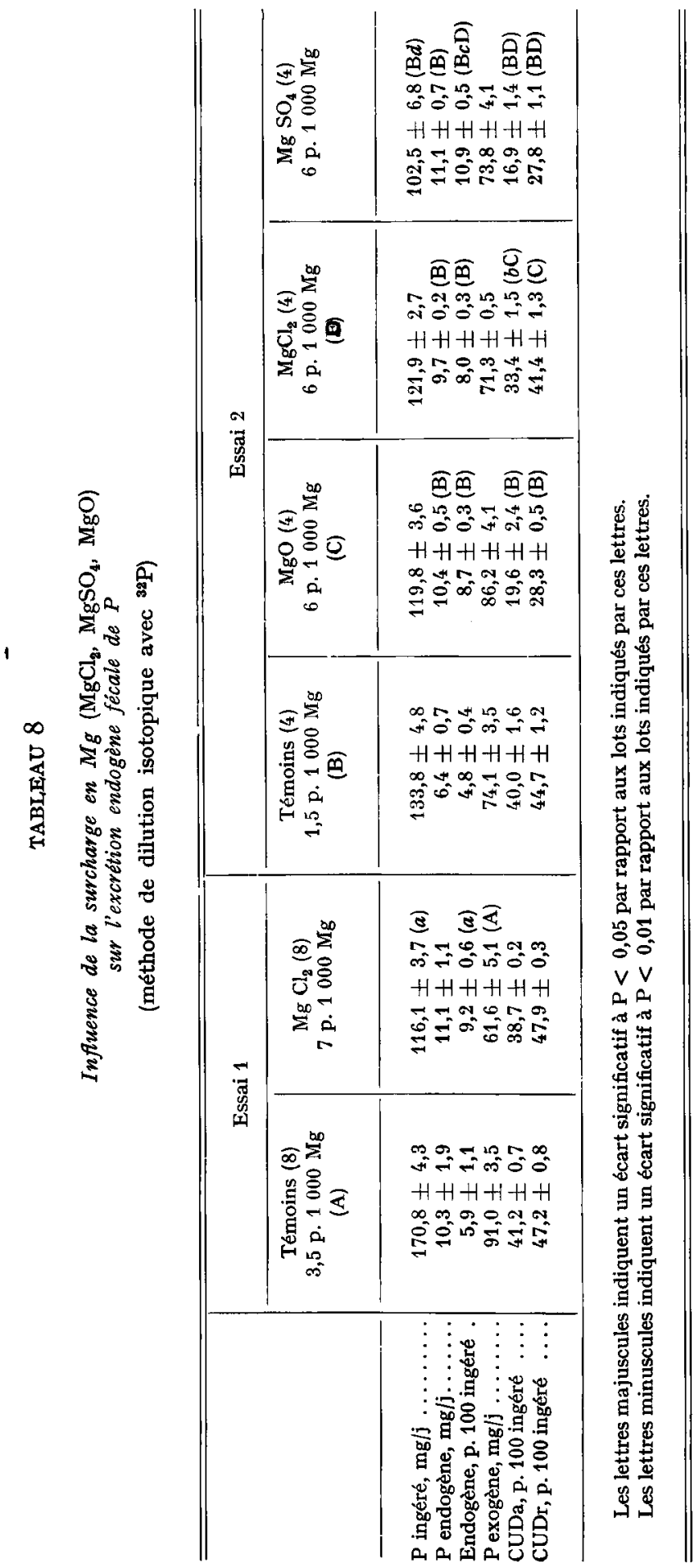


A. POINTILIART, L. GUÉGUEN

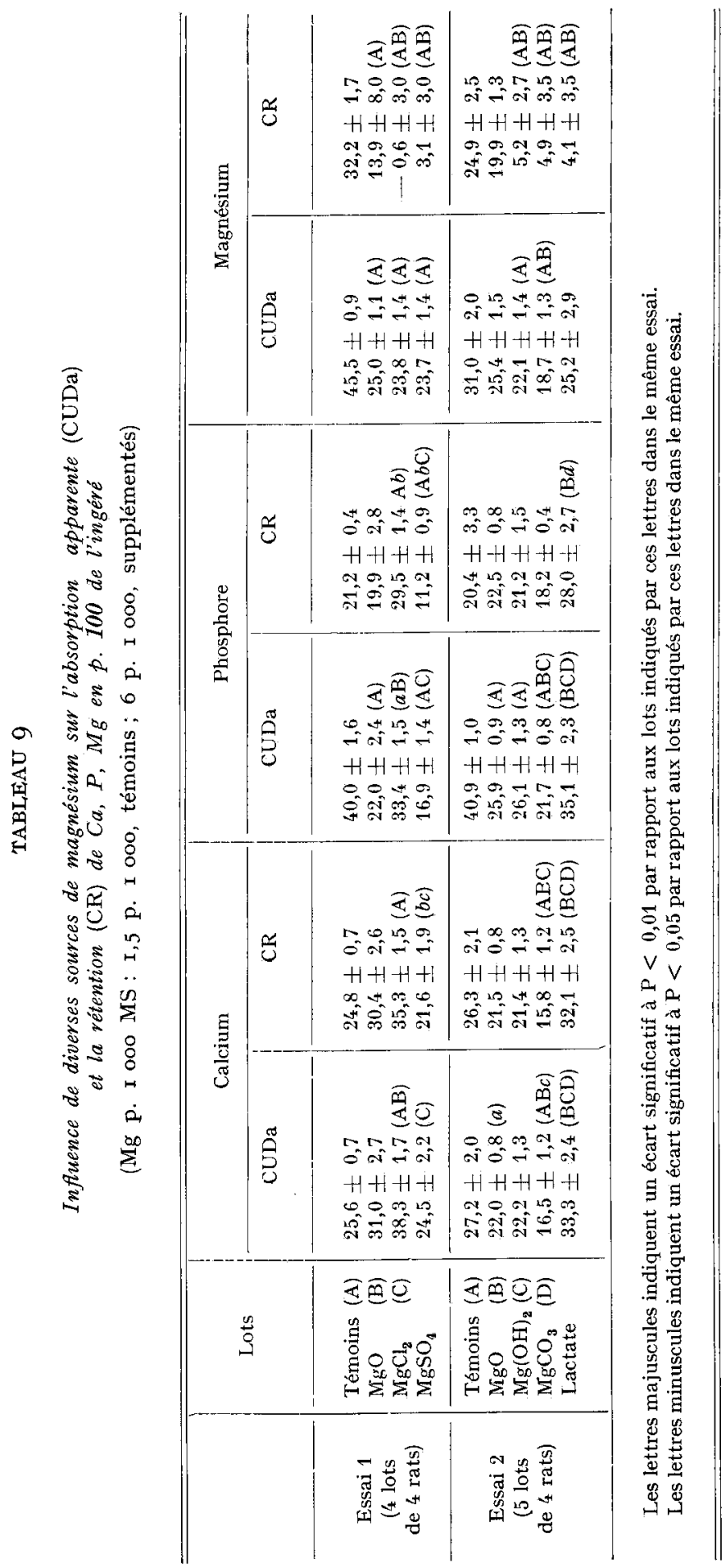


un supplément de carbonate de magnésium absorbent moins bien $\mathrm{Ca}$ et $\mathrm{P}$ que les rats ayant reçu l'hydroxyde, l'oxyde, le lactate, ou aucun supplément. Avec le lactate de magnésium, au contraire, les CUDa de $\mathrm{P}$ et de $\mathrm{Ca}$ sont supérieurs à ceux obtenus avec les autres sources de $\mathrm{Mg}$ sans être significativement différents de ceux des témoins.

Des essais I et 2, on peut déduire que, quelle que soit la source de Mg supplémentaire utilisée, on observe une baisse de l'absorption du phosphore en $\mathrm{p}$. Ioo de l'ingéré tandis que les effets sur l'absorption du calcium sont déterminés, en définitive, par la nature de l'anion.

En ce qui concerne la rétention, les résultats concernant le calcium sont comparables à ceux obtenus pour l'absorption, du fait de la faible importance de l'excrétion urinaire. Pour le phosphore, les rétentions enregistrées chez les rats supplémentés en $\mathrm{Mg}$ sont comparables à celles obtenues chez les témoins, sauf pour le chlorure de magnésium qui améliore la rétention et le sulfate qui la diminue.

Pour le magnésium, quelle que soit la source alimentaire utilisée, dans presque tous les cas (tabl. 9) le CUDa et le CR (coefficient de rétention) diminuent, sauf pour l'oxyde de magnésium dans un essai seulement (essai 2). Notons que le coefficient de rétention est négatif dans le cas du chlorure car les pertes urinaires sont très grandes avec ce sel (tabl. 5).

\section{DISCUSSION}

\section{A. - Effets urinaires de la supplémentation en magnésium}

L'effet constaté sur les phosphates concorde avec les travaux des autres auteurs qui ont étudié, chez le Rat, soit la carence en Mg (GrNn et Shanbour, r 967 ), soit l'excès apporté sous forme de chlorure (CLARK et BELANGER, I967; CLARK, I969). BRISCOE et RAGAN (I966) ayant également observé ce phénomène chez l'Homme, avec $\mathrm{Mg}(\mathrm{OH})_{2}$, il était intéressant de montrer que l'effet de $\mathrm{Mg}$ sur $\mathrm{P}$ urinaire s'exerçait non seulement avec ces formes d'apport mais aussi avec le sulfate, le lactate, l'oxyde et le carbonate de magnésium.. En ce qui concerne l'influence de $\mathrm{Mg}$ sur $\mathrm{Ca}$ urinaire, la nature de l'apport est déterminante. On peut observer, soit une hypercalciurie chez 1'Homme avec l'hydroxyde (Briscoe et RAGAN) et également le lactate (TIBETTS et AUB, I937), chez le Rat avec le chlorure, le sulfate et le citrate (RANDOIN et al., I952 ; BERTHAUX et al., I962 ; CLARK, I969), soit aucune modification significative chez le Rat avec le carbonate (Randorn et al.), chez le Mouton avec l'oxyde (ELLIOT et RIBEIRO, I97I ; MELNICK et al., I97I). Pour ce qui concerne le lactate et l'hydroxyde, nos résultats vont dans le même sens sans toutefois être significatifs. $\mathrm{La}$ comparaison des effets de $\mathrm{MgSO}_{4}$ sur $\mathrm{Ca}$ urinaire avec les effets rencontrés par les autres auteurs, soit avec $\mathrm{MgSO}_{4}$, soit avec $\mathrm{CaSO}_{4}$ (mais non avec $\mathrm{Na}_{2} \mathrm{SO}_{4}$ ) (Nutr. Rev., I957; BERTHAUX et al., I962; GuEGUEN et BESANÇON, I962) permet de penser à un effet propre de l'ion sulfate.

En examinant le résultat global (tabl. 9) de l'influence des différentes sources d'apport de $\mathrm{Mg}$, on constate que les formes basiques (carbonate, oxyde, hydroxyde et lactate) sont sans effet ou ont un effet négligeable sur la calciurie (lactate) com- 
parées aux formes acides (sulfate, chlorure). Or, en provoquant une acidose par des moyens divers (perfusion de $\mathrm{HCl}$, injection de sels acides comme $\mathrm{NH}_{4} \mathrm{Cl}$, respiration d'un excès de $\mathrm{CO}_{2}$ ) d'autres auteurs ont constaté chez le Rat (CLARK et GEOFrroy, I958) mais aussi chez le Mouton (STACY et WIISON, I970) une augmentation du calcium urinaire. On peut donc émettre l'hypothèse selon laquelle l'acidose, plutôt que la surcharge en $\mathrm{Mg}$, serait responsable de l'action au niveau rénal sur le calcium. Cependant, cela est controversé, car SzILAGYI (1970) a constaté que l'abaissement du $\mathrm{pH}$ sanguin provoquait chez le Chien, contrairement au Mouton, une diminution de $\mathrm{Ca}$ urinaire. D'autre part, chez le Chien encore, certains auteurs (SAMry et al., I960; MASSRY et al., 1970 ; MASSRY et KLEEMAN, 1970) ont mis en évidence, directement au niveau du rein, une compétition entre $\mathrm{Ca}^{++}$et $\mathrm{Mg}^{++}$, vis-à-vis d'un mécanisme commun de réabsorption tubulaire. Chez un rat au moins ayant reçu $\mathrm{MgO}$ sont apparus des calculs urinaires (vésicaux) qui posent un problème d'interprétation. D'après les différences entre les quantités de $\mathrm{Mg}$ et de $P$ respectivement retenues par les rats supposés formeurs de calculs et les rats non formeurs (tabl. 3) il semble que les calculs étaient à base de phosphates trimagnésiens, analogues à ceux obtenus dans l'urolithiase du Mouton (JEAN-BLAN et al., I968; EMERICK, I968). Or, EMFrick préconise l'apport de MgO dans la prévention des calculs urinaires chez 1'Agneau. Rappelons également que certains auteurs (MOORE et Bunce, I964 ; PrIen, I965 ; Albuguerque et Tuma, I962 ; Gershoff et PrIEN, I967) préconisent chez 1'Homme, contre 1'apparition de calculs non plus phosphatiques mais oxaliques, une thérapeutique magnésienne. De nouvelles investigations nous semblent donc nécessaires pour déterminer la responsabilité de la magnésie (MgO) dans l'apparition ou dans la prévention des calculs urinaires, d'autant que dans le deuxième essai (tabl 6) aucun cas de lithiase ne fut constaté. Il n'a pas non plus été constaté d'alcalinisation significative des urines, facteur reconnu déterminant dans la lithiase phosphatique du Mouton (EMERICK).

\section{B. - Effets du magnésium sur l'utilisation digestive du calcium et du phosphore}

L'absence d'influence de la surcharge en $\mathrm{Mg}$ sur les pertes endogènes fécales de $\mathrm{Ca}$ constatée chez le Rat, avait également été vue chez l'Homme par d'autres auteurs ayant employé des méthodes différentes (BRISCOE et RAGAN, I966; Mc CANCE; et WidDowson, I939). L'augmentation de l'excrétion endogène fécale de $P$ n'a pas été constatée, à notre connaissance, par d'autres auteurs. Il faut remarquer que l'augmentation de la perte endogène fécale de $\mathrm{P}$ est largement compensée par la conservation accrue de $\mathrm{P}$ au niveau rénal.

En ce qui concerne la surcharge de $\mathrm{MgCl}_{2}$ chez le Rat, les travaux de CLARK et de GoTOH et SEKI (I966) aboutissent à des résultats voisins des nôtres : l'excrétion fécale totale de calcium diminue tandis que celle de $\mathrm{P}$ augmente. Avec $\mathrm{MgCO}_{3}$, RANDorN et al. (1952) avaient également constaté, chez le Rat, une baisse de l'absorption du calcium sans constater pour autant de changement avec $\mathrm{MgSO}_{4}$ et $\mathrm{MgCl}_{2}$, comme dans cette expérience. Avec $\mathrm{Mg}(\mathrm{OH})_{2}$, BriscoE et RAGAN constatent une baisse de l'utilisation digestive du phosphore chez l'Homme tandis que chez le Mouton, MYBURGH et Du TOIT (I970) constatent seulement une baisse de celle du calcium avec $\mathrm{MgSO}_{4}$. Certains auteurs (ALCOCK et MCINTYRE, I962; SCHACHTER et ROSEN, 
I959; HENDRIx et al., I963) ont voulu mettre en évidence un phénomène de compétition entre $\mathrm{Ca}$ et $\mathrm{Mg}$ au niveau intestinal, mais il ne semble pas qu'il en soit ainsi dans cette expérience, au moins pour le chlorure, le sulfate et le lactate de magnésium. Dans un récent travail sur l'influence du magnésium dans le rachitisme du Rat, AYMARD et GounELLLE (I97I) concluent également à une absence de compétition entre le calcium et le magnésium au niveau intestinal. Le seul sel qui semble réduire nettement l'utilisation digestive du calcium est le carbonate de magnésium. Cela conduit à penser que, là encore, le $\mathrm{pH}$ jouerait vraisemblablement un rôle plus grand que la quantité de $\mathrm{Mg}$ présente dans la lumière intestinale. Par contre, il semble évident que le magnésium en excès dans le tube digestif gêne l'absorption des phosphates, peut être par la formation de phosphates magnésiens insolubles.

\section{C. - Effets du magnésium sur le rétention du calcium et du phosphore}

Dans la plupart des cas, 1a supplémentation en magnésium ne modifie pas la rétention du calcium $\left(\mathrm{MgO}, \mathrm{Mg}(\mathrm{OH})_{2}\right.$, lactate, $\left.\mathrm{MgSO}_{4}\right)$ ou la diminue $\left(\mathrm{MgCO}_{3}\right)$, seul un important supplément de chlorure pouvant l'améliorer. RANDonv et al. (I952) avaient noté les mêmes faits chez le Rat sans toutefois trouver de différence pour $\mathrm{MgCl}_{2}$.

Cr.ARK avait montré que, chez le Rat soumis à un régime enrichi en $\mathrm{MgCl}_{2}$, le bilan du phosphore (et du calcium) était amélioré. Il convient de noter qu'avec les autres sels, le bilan demeure inchangé, sauf avec $\mathrm{MgSO}_{4}$ où il diminue ( $\mathrm{C}_{4} \mathrm{ARK}$ et GEOFFroy, 1958) ce qui a aussi été observé chez le Mouton (MyBurgh et DuTort, I97I ; GUEGUEN et PoINTILLART, I973). Il faut signaler que de nombreux auteurs n'ont pas mis en évidence de relation entre le niveau de $\mathrm{Mg}$ ingéré et le $\mathrm{P}$ retenu, ni chez 1'Homme (LEISCHENRING et al., I95I), ni chez le Mouton (HJERPE, I968 a) ni chez le Rat (HAAG et PALMER, I928 ; GoTOH et SEKI, I966) pour des teneurs variées en $\mathrm{Ca}, \mathrm{P}, \mathrm{Mg}$ dans le régime.

En conclusion, que l'on considère le bilan global du calcium ou celui du phosphore, le rôle déterminant semble revenir à l'anion et non à la surcharge en $\mathrm{Mg}$. Les différences entre sels, quand elles apparaissent, sont quantitativement peu importantes, sauf dans le cas de $\mathrm{MgSO}_{4}$ qui provoque une chute de $50 \mathrm{p}$. 100 au moins de 1a rétention du phosphore par rapport aux autres lots (tabl. 9). Cependant, il importe de souligner que les observations que nous avons faites ne sont peut-être pas valables dans le cas d'un apport phospho-calcique moindre. Les interrelations $\mathrm{Ca}-\mathrm{P}-\mathrm{Mg}$ sont en effet complexes et il est possible que l'hypermagnésémie que nous avons provoquée mette en jeu des phénomènes endocriniens (LARVOR et DURLACH, I97I $a$ ).

Enfin, tant au niveau rénal qu'au niveau intestinal, les effets constatés dans certains de nos essais font intervenir, outre d'éventuels mécanismes de compétition entre électrolytes, des modifications de l'équilibre acido-basique qui nécessitent des investigations ultérieures. 


\section{SUMMARY}

\section{EFFECT OF LEVEL, AND NATURE OF MAGNESIUM IN THE DIET ON PHOSPHORUS, CALCIUM AND MAGNESIUM METABOLISM IN THE GROWING RAT}

Balance studies are done on one hundred growing rats receiving an excess of magnesium, either in variable quantity or in different chemical forms (chloride, sulphate, lactate, carbonate, anhydrous or hydrated oxide).

Endogenous fecal loss and true absorption of calcium and phosphorus are measured using radioisotopic methods - the comparative halance method (after oral or intraperitonal administration of a dose of ${ }^{45} \mathrm{Ca}$ ) for calcium, and the isotopic dilution method for phosphorus.

An excess of magnesium in any chemical form causes decrease of apparent absorption and urinary excretion of phosphorus, and in the form of chloride, sulphate and lactate, an increase of calcium urinary excretion. Intestinal absorption of calcium seems increased by the supplement of magnesium chloride and decreased by the supplement of magnesium carbonate.

Intestinal absorption and urinary excretion of calcium seem to vary more under the effect of anionic contribution and acido-basic balance than under the effect of magnesium supplementation. The supplement of magnesium oxide, $\mathrm{MgO}$, caused several cases of urolithiasis.

There does not seem to be any competition between magnesium and calcium in intestinal absorption.

\section{RÉFÉRENCES BIBLIOGRAPHIQUES}

Albuquerque P. F., de Tuma M., ig62. Investigations in urolithiasis. II. Studies on oxalate. $J$. Urol., 87,504 .

Alcock N., Mc Intyre I., 1962. Interrelation of calcium and magnesium absorption. Clin. Sci. 22, r65. ANoNyme I957. Magnesium and electrolyte excretion, clinical nutrition, Nutr. Rev. 15, 237.

Aymard P., Gounelle J. C., I97i. Le magnésium dans le rachitisme du Rat. C. R. Soc. Biol., 165, 2275-2279.

Berthaux P., Paupe J., Maurat J., I962. Action d'une surcharge orale en magnésium sur l'élimination urinaire du calcium chez le Rat blanc. C. R. Soc. Biol., 156, 51 .

Briscoe A. M., Ragan C., 1966. Effect of magnesium on calcium metabolism. Am. J. Clin. Nutr., $19,296$. Clark I., $1969 a$. Metabolism interrelations of Ca, Mg and P. Am. J. Physiol., 217, 87r.

ClARK I., I968 b. Importance of dietary $\mathrm{Ca}: \mathrm{PO}_{4}$ ratios on skeletal $\mathrm{Ca}, \mathrm{Mg}$ and $\mathrm{PO}_{4}$ and metabolism. Am. J. Physiol., 217, 865.

Clark I., 1969 c. Effect of $\mathrm{Mg}$ on $\mathrm{Ca}$ and $\mathrm{PO}_{4}$ metabolism in PTX rats. Endocrinology, 85, 1103.

Clark I., I968. The effects of $\mathrm{Mg}$ ions on $\mathrm{Ca}$ and $\mathrm{P}$ metabolism. Am. J. Physiol., 214, 348.

Clark I., 1968. Relation of $\mathrm{Mg}$ ions to $\mathrm{Ca}$ and $\mathrm{PO}_{4}$ absorption. Nature, 207, 982.

Clark I., Belanger L., I967. The effects of alterations in dietary $\mathrm{Mg}$ on $\mathrm{Ca}, \mathrm{PO}_{4}$ and skeletal metabolism. Calc. tiss. Res., 1, 204-218.

Clark I., Geoffroy R., r958. Studies in calcium metabolism. J. Biol. Chem., 233, 203.

Clark I., Rivera-Cordero F., I97I. Effect of parathyroid function on absorption and excretion of $\mathrm{Ca}_{\text {, }}$. $\mathrm{Mg}$ and $\mathrm{PO}_{4}$ by rats. Endocrinology, 88, 302-308.

Comar C. L., Monroe R. A., Visek W. J., Hansard S. L., I953. Comparison of two isotopic methods for determination of endogenous fecal calcium. J. Nutr., 50, 459.

Elliot J. S., Ribeiro M. E., I971. Magnesium and calcium oxalate solubility. Invest. Urol., 8, 386.

EMERICK R. J., I968. Causes and methods of prevention of phosphate urinary calculi in feed-lot lambs. Minnesota Nutr. Conf. Proceedings.

Gershoff S. N., Andrus S. B., r96r. Dietary $\mathrm{Mg}, \mathrm{Ca}$ and vitamin $\mathrm{B}_{6}$ and experimental néphropathies in rat : calcium oxalate calculi apatite nephrocalcinosis. $J$. Nutr., 73 , 308-316.

Ginn H. E., Shanbovr L. L., 1967. Phosphaturia in magnesium deficient rats. Am. J. Physiol., 212, I347.

Gотон S., SEKI H., r966. The effects of dietary magnesium level on calcium and phosphorus metabolism. Physiol. Bohemoslov., 15, 538 . 
Gut́guen L., I962. L'utilisation digestive du phosphore du foin de luzerne par le Mouton mesurée à l'aide de ${ }^{32} \mathrm{P}$. Ann. Biol. anim. Bioch. Biophys., 2, 143-149.

Gutéguen L., r963. Influence de la nature du régime alimentaire sur l'excrétion fécale de phosphore endogène chez le Veau. Ann. Biol. anim. Bioch. Biophys., 3, 243-253.

Gúfguen L., Besançon P., I972. Influence des sulfates sur le métabolisme phosphocalcique. I. Utilisation du sulfate de calcium par le Mouton. Ann. Biol. anim. Bioch. Biophys., 12, 589-598.

Gutguen L., Pointillart $Q$, I973. Influence des sulfates sur le métabolisme phosphocalcique. II. Influence comparée du soufre élémentaire, du sulfate de sodium et du sulfate de magnésium chez le Mouton. Ann. Biol. anim. Bioch. Biophys., (à paraître). .

HaAG J., Palmer L. S., I928. The effect of variation in the proportion of Ca, Mg and P contained in the diet. J. Biol. Chem., 76, 367-388.

Hansard S. L., Comar C. L., Plumlee M. P., I952. Absorption and tissue distribution of radiocalcium in cattle. J. Anim. Sci., 11, 524.

Hendrix J. Z., Alcock N. W., Archibald R. M., 1963. Competition between calcium, strontium and magnesium for absorption in the isolated rat intestine. Clin. Chem., 8, 734-744.

HJERPE C. A., I968. Influence of dietary magnesium on metabolism of calcium and magnesium in sheep. Am.J.Vet. Res., 29, 143-152.

Jean. Blain M., Jean-Blain A., Jean-Blain C., ig68. La sédimentose, un aspect de la lithiase urinaire des animaux de l'espèce ovine. Rev. Méd. vét., $519,624$.

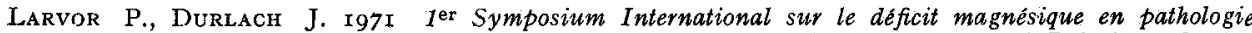
humaine, Vittel. a) Relations entre magnésium et glandes endocrines, p. 252-296. b) Relations physiologiques entre magnésium et nutrition, p. 297-325. Édition du Symposium, Jean Durlach, Paris (I97 I).

Leischenring J. M., Norris L. M., Lamison S. A., I95I. Magnesium metabolism in college women : observations on the effect of $\mathrm{Ca}$ and $\mathrm{P}$ in take levels. $J$. Nutr., $\mathbf{4 5}, 477$.

Lofgreen G. P., KLeiber M., I954. Further studies on the availability of phosphorus in alfalfa hay. J. Anim. Sci., 13, 258-264.

Massry S. G., Ahumada J. J., Coburn J. W., Kleeman C. R., x97o. Effect of magnesium loading on renal excretion of calcium and sodium during reduction in their filtered loads. Clin. Res., 17, 150.

MassRy S. G., KLEeman C. R., I97o. The excretion of calcium and magnesium during acute rise in glomerular filtration rate in the presence or absence of extracellular volume expansion. Clin. Res., 18, 195 .

Mc CANCE R. A., Widdowson E. M., I939. The fate of calcium and magnesium after intraveinous administration to normal persons. Biochem. J., 33, 523 .

Melnick I., Landes R. R., Hoffman A. A., Burch J. F., r971. Magnesium therapy for recurring calcium oxalate urinary calculi. J. Urol, 89 .

Moore C. A., Bunce G. E., I 964 . Reduction in frequency of renal calcium formation by oral magnesium administration. Invest. Urol., 2, 7 .

Myburgh S. J., Dutoit I. de V., I97o. The influence of $\mathrm{MgSO}_{4}$ on the absorption, excretion and retention of $\mathrm{Ca}$ and $\mathrm{P}$ by sheep fed on phosphate supplemented rations. Ondersteport J. Vet. Res., 37, I6.

Patterson M. S., Greene R. C., I965. Measurement of low energy beta-emitters in aqueous solution by liquid scintillation counting of emulsions. Anal. Chem., 37, 854 .

Pointillart A., Ig7I. Les interactions du calcium, du phosphore et du magnésium. Conséquences nutritionnelles et endocriniennes. Th. Doct. vét., I97I (Alfort).

Prien E. L., 1965. Renal calculi and oral magnesium therapy. J.A. M. A., 192, x77.

Randoin L., Causeret J., Hugot D., Morel G., 1952. Influence de divers sels magnésiens administrés par voie orale sur la rétention du calcium daus l'organisme. Bull. Soc. Chim. Biol., 34, I I59.

Saminy H. H., Brown J. L., Globus D. L., Kessler R. H., Thompson D. D., I96o, Interrelations between renal transport system of magnesium and calcium. Amer. J. Physiol., 198, 599.

Schachter D., Rosen S. M., 1959 Active transport of ${ }^{45} \mathrm{Ca}$ by the small intestine and its dependance on vitamine D. Amer. J. Physiol., 196, 357-362.

Stacy B. O., Wilson B. W., I97o. Acidosis and hypercalciuria renal mechanisms affecting Ca-Mg and $\mathrm{Na}$ excretions in the sheep. J. Physiol., 210, 549-564.

SzILAGYI G., I970. Effect of acidification and alcalinization on calcium and citrate metabolism in normal and parathyroidectomized dogs. Acta Physiol., 38, 281-290.

Tibetrs D. M., Aub J. C., I937. Magnesium metabolism in health and disease. I. The Mg and Ca excretion of normal individuals. Also the effect of $\mathrm{Mg}$, chloride and phosphate ions. $J$. Clin. Invest., 16, 49I. 